$$
\text { CONF- } 960 / 63-20
$$

LA-UR-96- 640

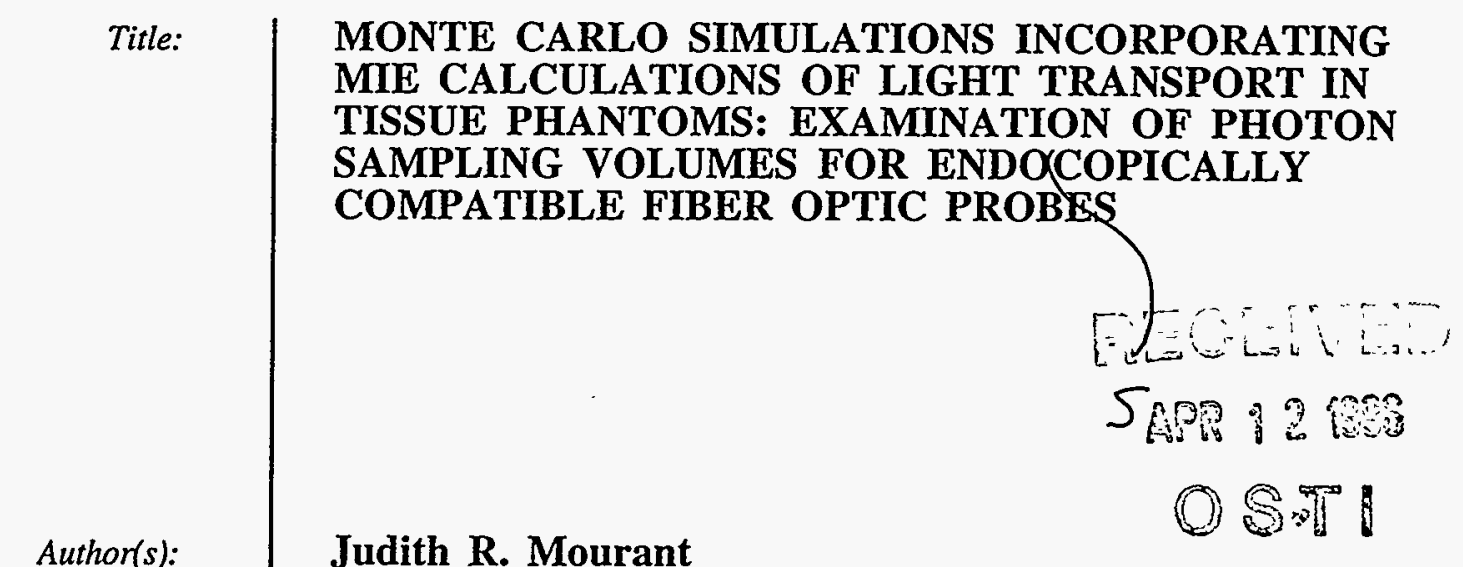

Submitted to: $\quad$ SPIE Biomedical Optics Conference 01/27/96-02/01/96

San Jose, CA

Andreas H. Hielscher

Irving J. Bigio

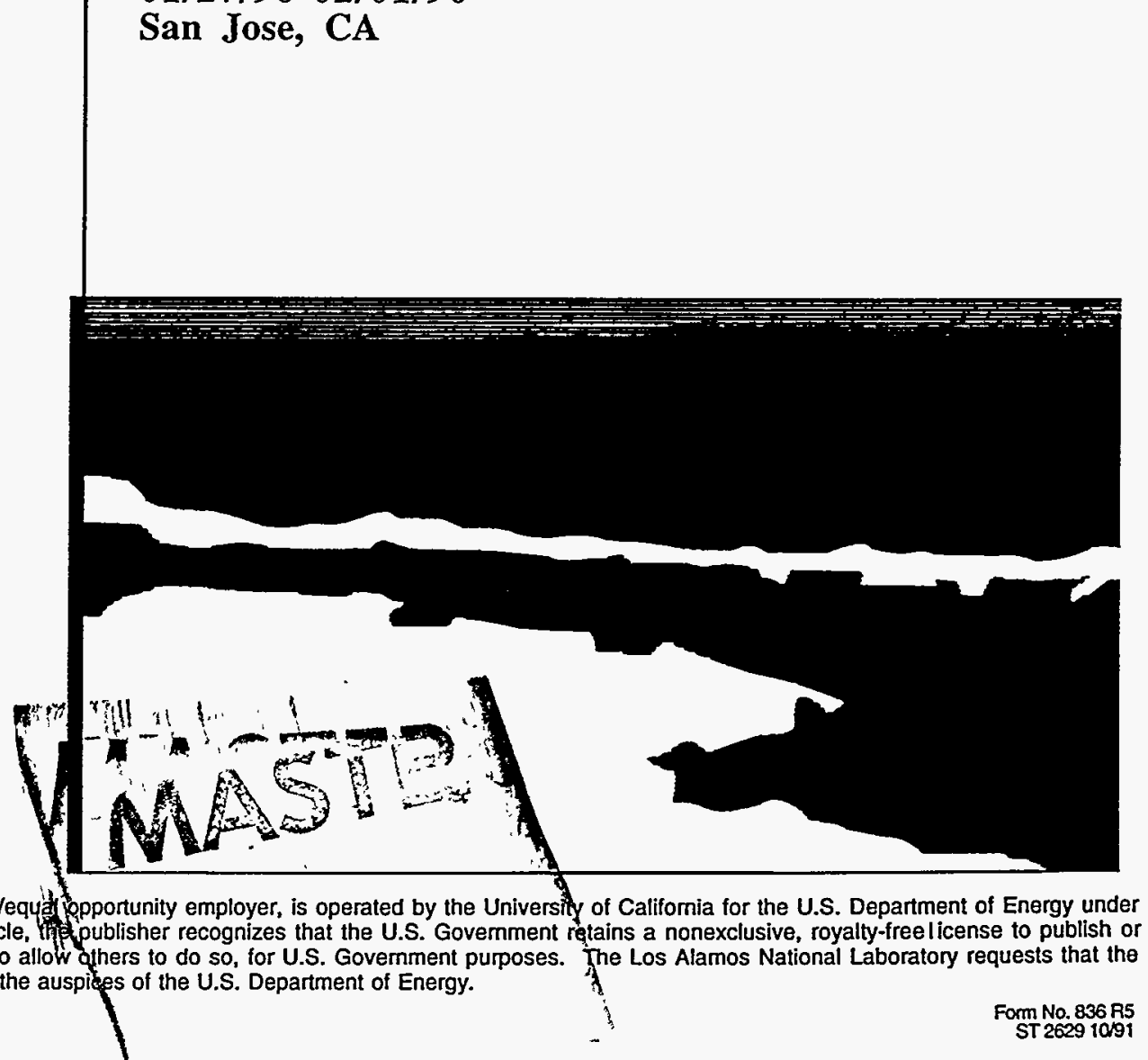




\title{
Monte Carlo Simulations incorporating Mie calculations of Light Transport in Tissue Phantoms: Examination of photon sampling volumes for endoscopically compatible fiber optic probes
}

\author{
Judith R. Mourant, Andreas H. Hielscher, and Irving J. Bigio \\ CST-4, Bioscience and Biotechnology Group, MS E535 \\ Los Alamos National Laboratory \\ Los Alamos, NM 87545
}

\section{ABSTRACT}

Details of the interaction of photons with tissue phantoms are elucidated using Monte Carlo simulations. In particular, photon sampling volumes and photon pathlengths are determined for a variety of scattering and absorption parameters. The Monte Carlo simulations are specifically designed to model light delivery and collection geometries relevant to clinical applications of optical biopsy techniques. The Monte Carlo simulations assume that light is delivered and collected by two, nearly-adjacent optical fibers and take into account the numerical aperture of the fibers as well as reflectance and refraction at interfaces between different media. To determine the validity of the Monte Carlo simulations for modeling the interactions between the photons and the tissue phantom in these geometries, the simulations were compared to measurements of aqueous suspensions of polystyrene microspheres in the wavelength range $450-750 \mathrm{~nm}$.

Key Words: Monte Carlo, Mie, elastic-scatter, photon sampling volume, photon pathlength

\section{INTRODUCTION}

In many applications of optics to tissue diagnostics it is important to know where in the tissue the collected light traveled (the photon sampling volume) and to understand how parameters of the light delivery and collection fibers may affect the photon sampling volume. In particular we are interested in the case where light delivery and collection is via optical fibers in close proximity to each other. This geometry is very relevant to clinical applications, because many implementations of optical techniques for tissue diagnosis require that the probe fit through a small aperture, such as the working channel of an endoscope, thus requiring adjacent or nearly adjacent delivery and collection fibers.

To examine light transport under these conditions Monte Carlo simulations are employed. We begin by modeling light transport in a well characterized tissue phantom - a suspension of polystyrene spheres. Wavelength dependent calculations of photon transport are compared with wavelength dependent measurements of light transport in these suspensions in order to determine the accuracy of the Monte Carlo simulations. Subsequently, photon sampling volumes and pathlengths are calculated for a variety of values for the scattering coefficient, $\mu_{\mathrm{S}}$, the absorption coefficient, $\mu_{\mathrm{a}}$, and the phase function, $\mathrm{P}(\theta)$, as well as for different fiber geometries.

3. MATERIALS AND METHODS

\subsection{Monte Carlo simulations}

Monte Carlo simulations were performed on a DEC Alphastation 250 4/266 in C++. The length of each photon step is $-\ln \zeta /\left(\mu_{S}+\mu_{\mathrm{a}}\right)$, where $\zeta$ is a random number, in the interval $(0,1]$, and for each step the photon weight is decreased by $d w=\mu_{a} /\left(\mu_{\mathrm{a}}+\mu_{\mathrm{S}}\right){ }^{1}$ Our code is specifically designed for modeling photon transport when optical fibers in close proximity to each other are used for light delivery and collection, and for modeling the wavelength dependence of the collected light. The geometry for the simulations is shown in Fig. 1. The numerical aperture of the fibers is accounted for both in light delivery and collection. Wavelength dependent light transport parameters, $\mu_{\mathrm{a}}(\lambda), \mu_{\mathrm{s}}(\lambda)$ and $\mathrm{P}(\theta, \lambda)$ are calculated using Mie theory. The parameters used for the Mie calculations are the radius of the polystyrene spheres, and the wavelength dependent indices of refraction for polystyrene, water, and quartz. Refractive indices of 
water, polystyrene and quartz from 450 to $800 \mathrm{~nm}$ were extrapolated from literature values. $2,3,4$ Reflection and refraction at the phantom surface are calculated as a function of wavelength. Photons are terminated either when they leave the semi-infinite medium or when they reach a distance from the fibers such that the likelihood of the photon returning to the collection fiber is negligible. The use of these criteria for photon termination was required rather than the more common method of terminating a photon after its weight goes below a certain value, because some of the simulations were run with no absorption.

One question we wish to address is: Where in the tissue phantom did the collected photons travel? One method for addressing this question is to store on a grid the locations where collected photons interacted with the medium on their path from the delivery to the collection fiber. A grid was defined in the $\mathrm{x}-\mathrm{z}$ plane. As each photon was propagated in the Monte Carlo simulation, the location of each interaction with the scattering medium was recorded in a temporary 2-D array. (The indices of this array are coordinates along the $\mathrm{x}$ and $\mathrm{z}$ axes.) When the photon is terminated the locations of the interactions, multiplied by the fraction of the photon which was deposited in the collection fiber, were added to a permanent 2-D array of. interaction locations. This is similar to the method used by Okada et. al. 5

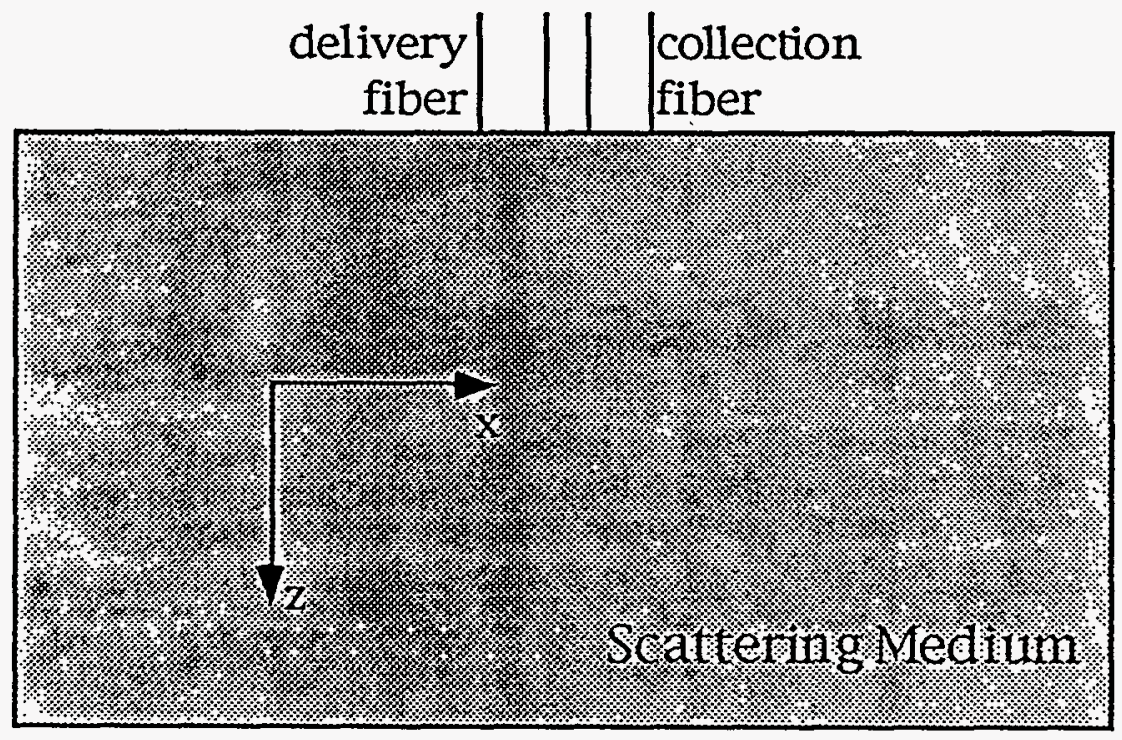

Fig. 1 Geometry for the Monte Carlo simulations and elastic-scatter measurements.

\subsection{Measurements of elastic-scatter in suspensions of polystyrene spheres}

Suspensions of polystyrene spheres were used as tissue phantoms. Known concentrations of polystyrene ! spheres were placed in sealed bottles to avoid chances in concentration due to evaporation and a small portion of the lid was cut out and replaced with mylar for light delivery. A 400 micron (0.22 NA) optical fiber was used for light delivery and light was collected by a 600 micron ( $0.48 \mathrm{NA}$ ) optical fiber. Both : fibers were placed directly on the mylar with a center-center separation of 550 microns. The mylar prevented any problems with meniscus forming around the fibers. The elastic-scatter signal was measured as $I$ (spheres)/I(ref), where $I$ (spheres) is the signal from the spheres and I(ref) is the signal from a diffuse reflector with a flat spectral response over the range of interest, $450-750 \mathrm{~nm}$. Thus the spectral response of the measurement system was accounted for. No attempt was made to calibrate the absolute value of the measured signal. Only the wavelength dependence of the measurements was recorded.

\section{RESULTS}

\subsection{Comparison of Monte Carlo results and elastic-scatter measurements}

Before using a Monte Carlo simulation to make predictions about photon transport, it is important to demonstrate that the code accurately simulates the physical situation. Although Monte Carlo simulations 
have been used extensively in modeling photon transport in tissue, very few comparisons of experimental and Monte Carlo results have been made for measurement geometries where the source and detector separation is small. ${ }^{6}$ The experiments described in the materials and methods section are more sensitive to both the phase function, $\mathrm{P}(\theta)$, and attributes of the light delivery and collection geometry than measurements performed with large source to detector separations or measurements made in more absorbing media. We have shown in a previous publication that our Monte Carlo simulations can reproduce the wavelength dependence of scattering in a medium where the wavelength dependence of absorption is strong. ${ }^{7}$ A comparison of Monte Carlo simulations with measurements on suspensions of $0.966 \mu \mathrm{m}$ and $3.0 \mu \mathrm{m}$ diameter polystyrene microspheres is shown in Fig. 2 . The concentration of the spheres was such that $\mu_{\mathrm{s}}$ ' was in the range $13-16 \mathrm{~cm}^{-1}$ from $450-750 \mathrm{~nm}$ for both sphere sizes. The wavelength dependence of the Monte Carlo simulations agrees reasonably well with the wavelength dependence of the measurements although there are some discrepancies particularly for the 0.966 micron diameter spheres.

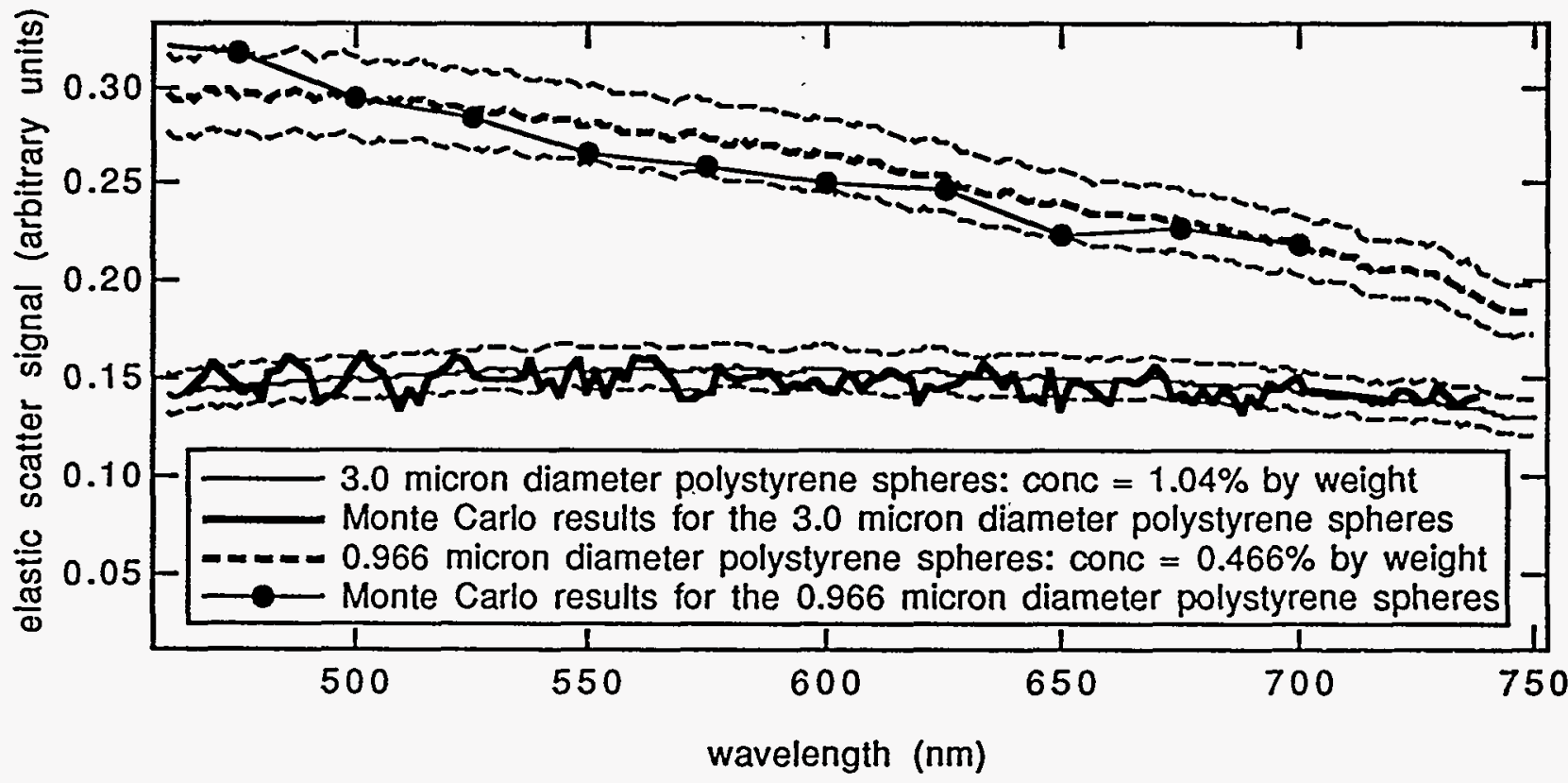

Fig. 2. The wavelength dependence of the elastic-scatter signal of suspensions of polystyrene spheres compared with wavelength dependent Monte Carlo simulations of light transport in the same media. The dashed lines are errors on the experimental curves estimated from repeated measurements. The errors on the Monte Carlo simulation results are roughly \pm 0.01 .

\subsection{Photon sampling volumes}

Photon sampling volumes are expected to depend on scattering and absorption parameters as well as on the measurement geometry. Intuitively one expects that the photon sampling volume is not as deep for greater absorption. This was found to be true as is shown in the top two plots of Fig. 3, where the number of interactions per bin, per collected photon is plotted as a function of $\mathrm{x}$ and $\mathrm{z}$ for 400 micron light delivery and collection fibers. On the left, where there is no absorption, the photons penetrate more deeply and the photon sampling volume is broader than on the right where $\mu_{\mathrm{a}}=2.7 \mathrm{~cm}^{-1}$. The bottom two plots of Fig. 3 are photon sampling volumes which compare the how the medium is sampled when 400 micron fibers are used for light delivery and collection versus when 200 micron fibers are used. When the 200 micron fibers are used a much narrower volume is sampled and light does not penetrate quite as deeply. For all plots, the grid size was 50 microns and the numerical aperture of the fibers was 0.48 . The scattering parameters for the simulations are calculated for 3.0 micron diameter polystyrene microspheres at $400 \mathrm{~nm}$ for a sphere concentration of $1.04 \%$ by weight at $400 \mathrm{~nm}$ giving $\mu_{\mathrm{s}}=94.5 \mathrm{~cm}^{-1}$ and $\mathrm{g}=0.8676$. (The two plots on the left in Fig. 3 are the same data plotted on different scales.) 

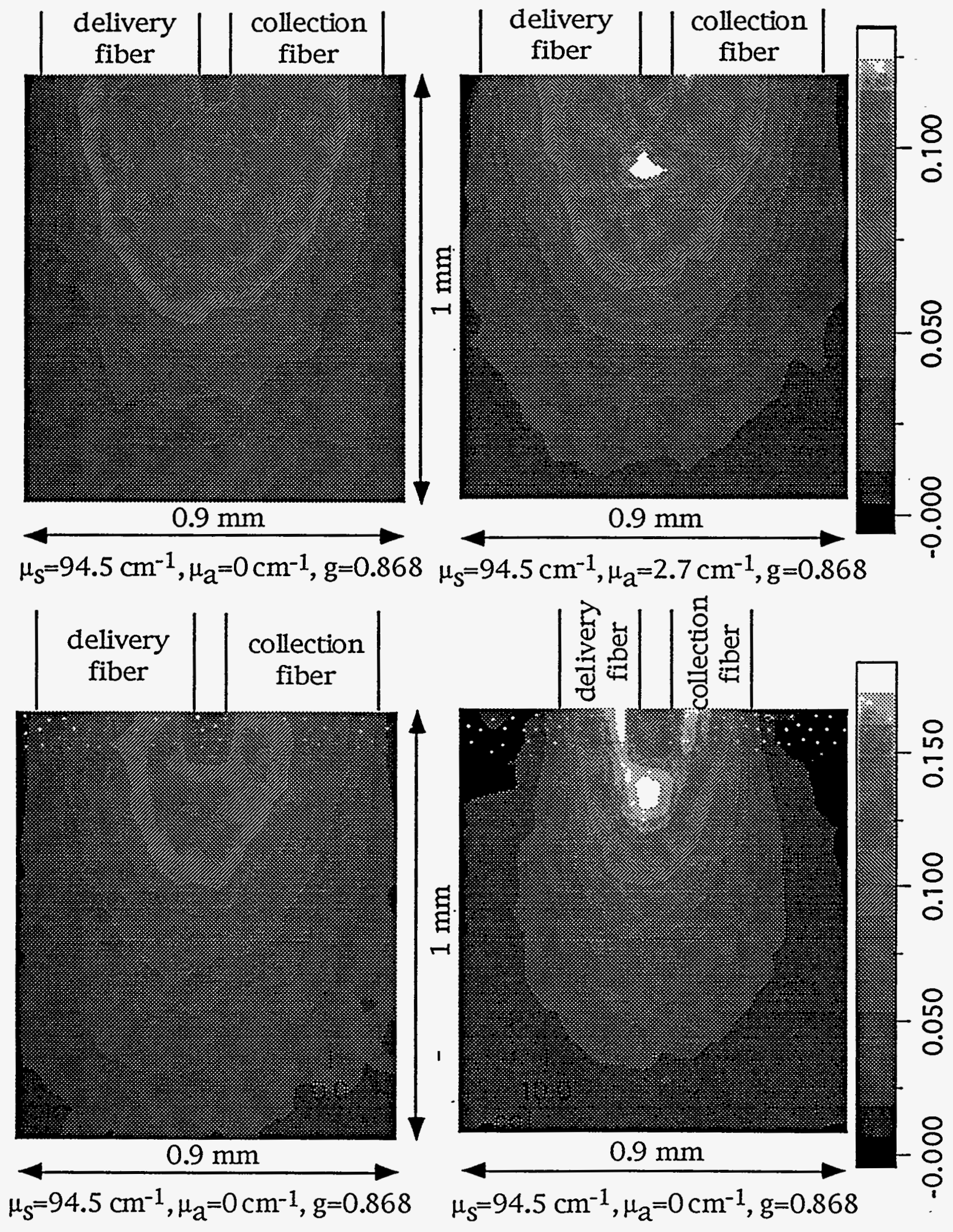

Fig. 3 See text for details. 


\subsection{Depth of photon penetration and photon pathlengths}

The depth profiles for the photon sampling volumes shown in Fig. 4 a were obtained by integrating the photon sampling volume over the $\mathrm{x}$ axis. Fig. 4 is a plot of the number of interactions at a given depth per bin per collected photon for $\mu_{S}=94.5 \mathrm{~cm}^{-1}, \mathrm{~g}=0$ and $\mu_{\mathrm{a}}=0$ to $2.7 \mathrm{~cm}^{-1}$. As expected the depth of the medium which is sampled decreases as absorption is increased. The photon pathlength also decreases with absorption in the medium as is shown in Fig. 4b. Fig. 5 gives the depth profile of the photon sampling volume for several different scattering parameters. As $g$ increases the photon sampling volume becomes less localized near the surface and penetration is deeper. The fraction of the collected photons which penetrated more than $0.5 \mathrm{~mm}$ into the medium is also deeper when the scattering coefficient is decreased. Although not shown, changes in the scattering phase function will also affect the average pathlength of the collected photons. As $\mathrm{g}$ increases from 0.8262 to 0.9142 , the average pathlength changes from 3.59 to $3.74 \mathrm{~mm}\left(\mu_{\mathrm{S}}=94.5 \mathrm{~cm}^{-1}\right.$ and $\left.\mu_{\mathrm{a}}=0\right)$. As $\mu_{\mathrm{s}}$ increases from 70.9 to $126.3 \mathrm{~cm}^{-1}$, the average pathlength changes from 3.81 to $3.66 \mathrm{~mm}\left(\mu_{\mathrm{a}}=0\right.$ and $\left.\mathrm{g}=0\right)$.
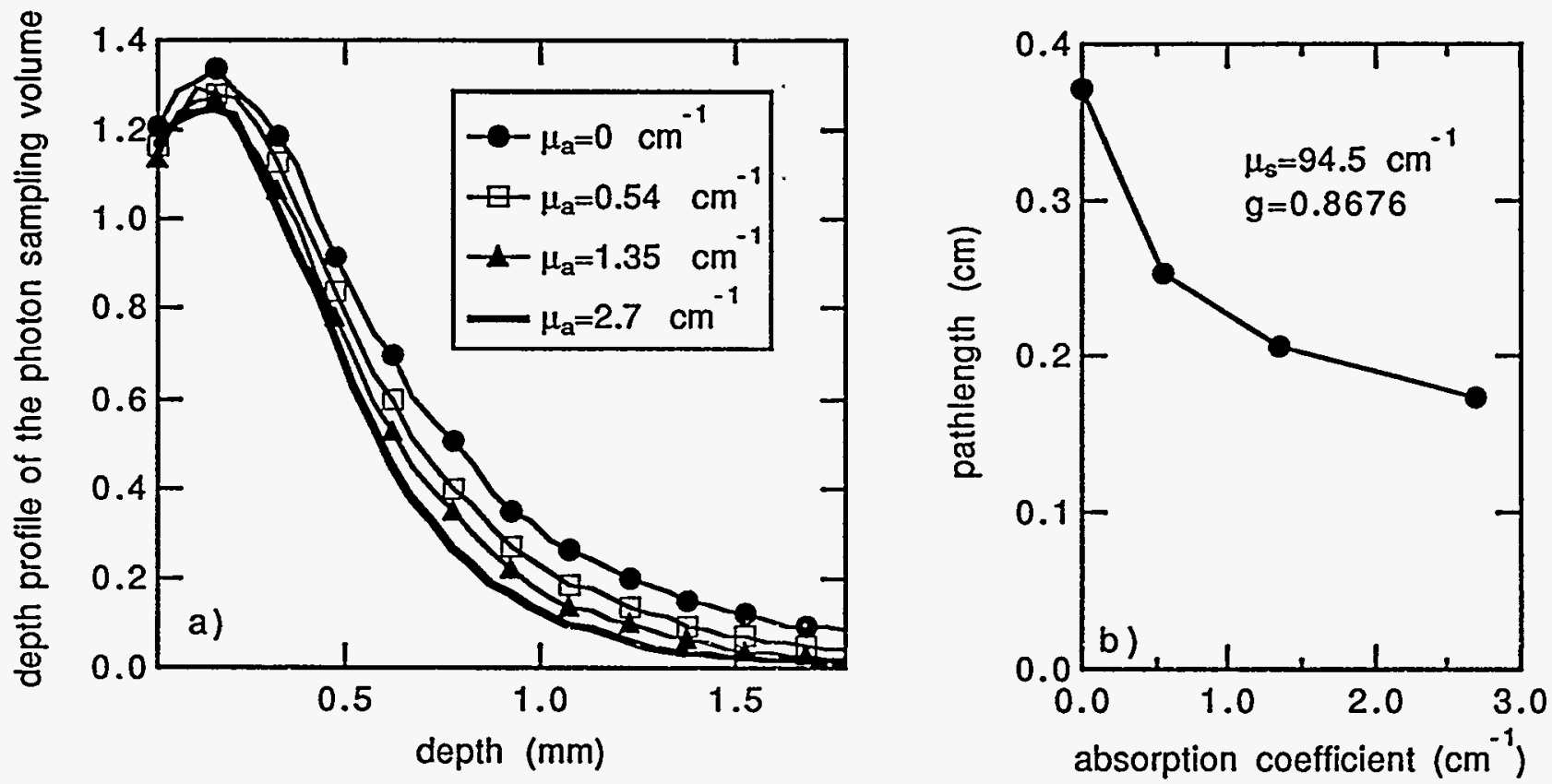

Fig. 4. a) Depth profile of the photon sampling volume. $\mu_{S}=94.5 \mathrm{~cm}^{-1}, \mathrm{~g}=0.8676$. b) Pathlength of the collected photons as a function of absorption. The collection and delivery fibers were both 400 microns in diameter with numerical apertures of 0.48 . The fiber separation was 550 microns.

\subsection{DISCUSSION AND CONCLUSIONS}

Fluorescence and elastic-scatter spectroscopy are being developed for diagnosis of tissue pathologies in several organs which are endoscopically accessible including the bladder, colon, and esophagus.8,9,10 Most cancers in these organs originate from epithelial tissue. Therefore, it is important that optical techniques probe the epithelial tissue, although for some applications it may be useful to probe deeper. Changes in fluorescence in the colon have been attributed to changes in the thickness of the mucosa which can only be noticed if the optical technique is probing into the submucosa. In general, the mucosa of the colon and esophagus is $\sim 1 \mathrm{~mm}$ or less thick, while in the bladder it is $<10$ cell layers thick. The results in Figs. 3, 4, and 5 demonstrate that an optical probe, using adjacent 400 micron diameter fibers with numerical apertures of 0.48 , will probe roughly one millimeter into a scattering medium with optical properties similar to tissue. The exact depth that is probed depends on the absorption, scattering and 
scattering anisotropy. For the range of optical parameters found in tissue we found that the tissue optical parameter that has the greatest effect on the depth of the photon sampling volume is absorption.

!
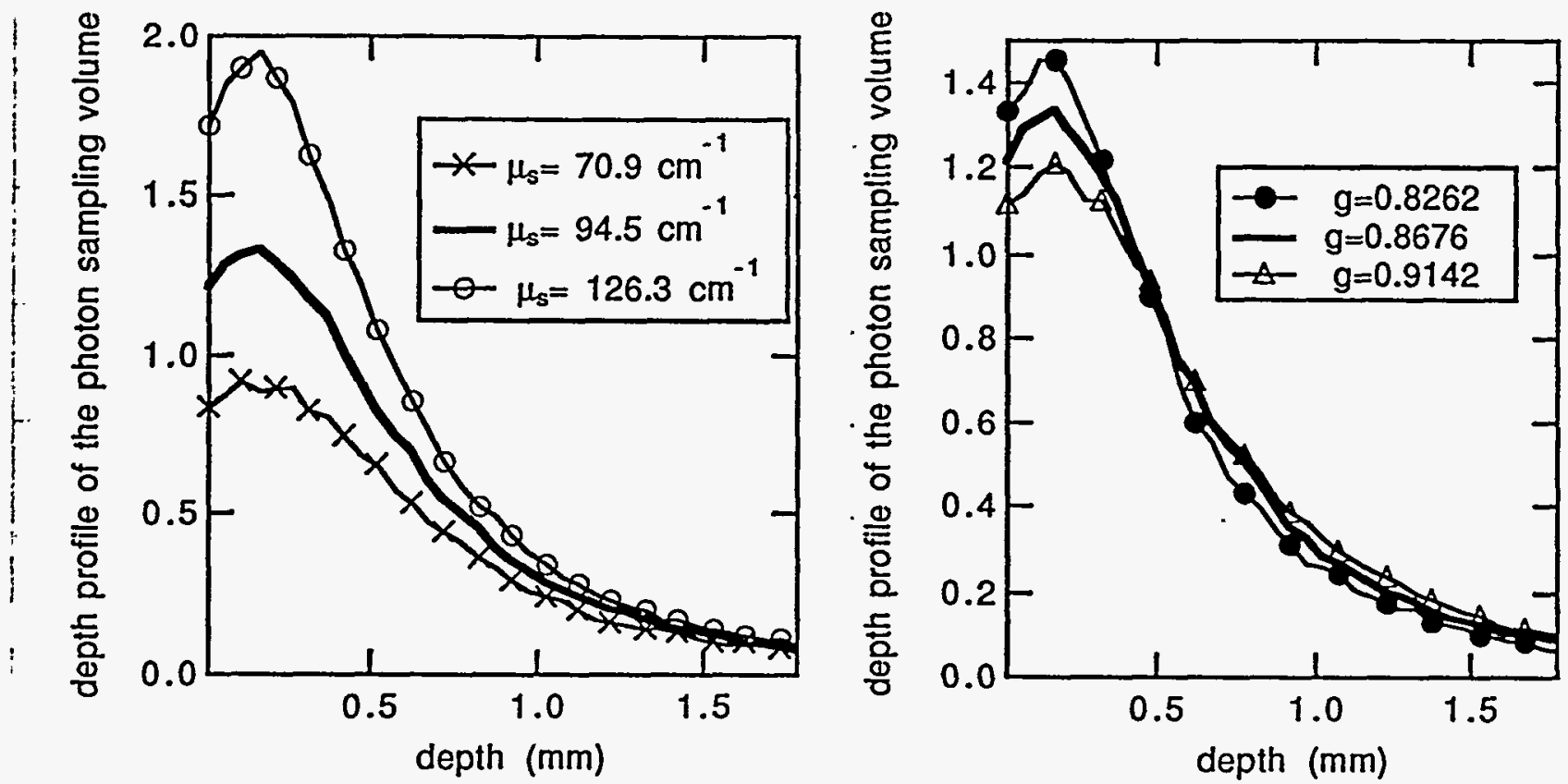

Fig. 5 a) Depth profile of the photon sampling volume for different values of scattering coefficient. For all curves $\mu_{\mathrm{a}}=0 \mathrm{~cm}^{-1}, \mathrm{~g}=0.8676$. b) Depth profile of the photon sampling volume for different $\mathrm{g}$ values. For all curves $\mu_{\mathrm{a}}=0 \mathrm{~cm}^{-1}$. and $\mu_{\mathrm{s}}=94.5 \mathrm{~cm}^{-1}$

In this paper we have studied how properties of scattering media, $\mu_{\mathrm{a}}, \mu_{\mathrm{s}}$ ' and $\mathrm{g}$, affect the photon sampling volume. For the small fiber separations addressed, it may not be possible to characterize the scattering anisotropy using only $g$. The shape of, the phase function, $P(\theta)$, may also affect the transport : of photons. This will be addressed in a separate paper. Several parameters of the light delivery and : collection system will also affect the photon sampling volume. In Fig. 3 it was shown that using 200 : micron fibers instead of 400 micron fibers greatly reduces the width of sampled region and slightly reduces the depth of the sampled region. Fiber separation will also affect the photon sampling volume and : preliminary results show that numerical aperture can significantly affect the photon sampling volume. Both of these issues will be addressed in later papers.

\subsection{ACKNOWLEDGEMENTS}

JoAnne Lacey and Tamara M. Johnoson contributed to and assisted in the measurements of the 1 suspensions of the polystyrene spheres

\subsection{REFERENCES}

1. L. H. Wang, S. L. Jacques, and L. Zheng, "MCML - Monte Carlo modeling of light transport in multi-layered tissues" Computer Methods and Programs in Biomedicine 47: 131-146 (1995).

2. Melchor Centeno V "The refractive index of liquid water in the near infrar-red spectrum" JOSA 31:244-2447 (1941). 
3. C. Tribastone and C. Teyssier "Designing plastics optics for manufacturing" Photonics Spectra May 1991 pg120-128.

4. CRC Handbook of Chemistry and Physics, 65th edition, R. C. Weast ed. (CRC, Cleveland, Ohio, 1984).

5. E. Okada, M. Firbank, and D. T. Delpy "The effect of overlying tissue on the spatial sensitivity profile of near-infrared spectroscopy 40: 2093-2108 (1995).

6 S. T. Flock, B. C. Wilson, and M. S. Patterson, "Monte Carlo modeling of light propagation in highly scattering tissues - II. Comparison with measurements in phantoms" IEEE Trans. Biomed. Eng. 36(12):1169-1173.

7. J. Boyer, J. R. Mourant and I. J. Bigio."Theoretical and experimental investigations of elastic scattering spectroscopy as a potential diagnostic for tissue pathologies." Optical Imaging and Photon Migration - OSA proceedings 21 (1994) 265-268.

8. J. R. Mourant, Irving J. Bigio, James Boyer, Richard L. Conn, Tamara Johnson, and Tsotomu Shimada, "Spectroscopic Diagnosis of Bladder Cancer with Elastic Light Scattering," Lasers Surg. Med. 17: 350-357 (1995).

9. K. T. Schomacker, J. K. Frisoli, C. C. Compton, T. J. Flotte, J. M. Richter, N. S. Nishioka, and T. M. Deutsch, "Ultraviolet laser-induced fluorescence of colonic tissue: basic biology and diagnostic potential," Lasers Surg. Med. 12: 63-78 (1992).

10. T. Vo-Dinh, M. Panjehpour, B. F. Overholt, C. Farris, F. P. Buckley III, and Rick Sneed, "In vivo cancer diagnosis of the esophagus using differential normalized fluorescence (DNF) indices, "Lasers Surg. Med. 16: $41-47$ (1995).

\section{DISCLAIMER}

This report was prepared as an account of work sponsored by an agency of the United States Government. Neither the United States Government nor any agency thereof, nor any of their Government. Neither the United States Governmed, or assumes any legal liability or responsiemployees, makes any warranty, express or implied, or assumes any legal liapparatus, product, or bility for the accuracy, completeness, or usefulness of any information, appowned rights. Referprocess disclosed, or represents that its use would not infringe privately owned re rame, trademark, ence herein to any specific commercial product, process, or serice imply its endorsement, recommanufacturer, or otherwise does not necessarily constitute or imply its endors thereof. The views mendation, or favoring by the United States Government or any agency therest Those of the

and opinions of authors expressed herein do not
United States Government or any agency thereof. 